TITLE:

\title{
Landau-Peierls instability in a Fulde-Ferrell type inhomogeneous chiral condensed phase
}

\section{AUTHOR(S):}

Lee, Tong-Gyu; Nakano, Eiji; Tsue, Yasuhiko;

Tatsumi, Toshitaka; Friman, Bengt

\section{CITATION:}

Lee, Tong-Gyu ...[et al]. Landau-Peierls instability in a Fulde-Ferrell type inhomogeneous chiral condensed phase. Physical Review D 2015, 92(3): 034024.

ISSUE DATE:

2015-08-27

URL:

http://hdl.handle.net/2433/201977

RIGHT:

(c) 2015 American Physical Society. 


\title{
Landau-Peierls instability in a Fulde-Ferrell type inhomogeneous chiral condensed phase
}

\author{
Tong-Gyu Lee* \\ Yukawa Institute for Theoretical Physics, Kyoto University, Kyoto 606-8502, Japan and Department of \\ Physics, Kyoto University, Kyoto 606-8502, Japan \\ Eiji Nakano ${ }^{\dagger}$ and Yasuhiko Tsue \\ Department of Physics, Kochi University, Kochi 780-8520, Japan \\ Toshitaka Tatsumi ${ }^{\S}$ \\ Department of Physics, Kyoto University, Kyoto 606-8502, Japan \\ Bengt Friman \\ GSI, Helmholzzentrum für Schwerionenforschung, Planckstrasse 1, D-64291 Darmstadt, Germany
}

(Received 16 April 2015; published 27 August 2015)

\begin{abstract}
We investigate the stability of an inhomogeneous chiral condensed phase against low energy fluctuations about a spatially modulated order parameter. This phase corresponds to the so-called dual chiral density wave in the context of quark matter, where the chiral condensate is spatially modulated with a finite wave vector in a single direction. From the symmetry viewpoint, the phase realizes a locking of flavor and translational symmetries. Starting with a Landau-Ginzburg-Wilson effective Lagrangian, we find that the associated Nambu-Goldstone modes, whose dispersion relations are spatially anisotropic and soft in the direction normal to the wave vector of the modulation, wash out the long-range order at finite temperatures, but support algebraically decaying long-range correlations. This implies that the phase can exhibit a quasione-dimensional order as in liquid crystals.
\end{abstract}

DOI: $10.1103 /$ PhysRevD.92.034024

PACS numbers: 21.65.Qr, 12.39.Fe

\section{INTRODUCTION}

Unveiling the phase diagram of quantum chromodynamics (QCD) is among the most fundamental issues in quarkhadron physics. So far, considerable theoretical and experimental efforts have been devoted to exploring the QCD phase diagram [1]; the properties of the high temperature regime are studied experimentally in ultrarelativistic heavy-ion collisions, and in $a b$ initio lattice QCD simulations. The latter are subject to technical difficulties at nonzero net baryon-number density, the so-called sign problem. In the near future, data at lower beam energies, relevant for the exploration of the phase diagram at nonvanishing baryon density, will be forthcoming. In order to exploit this opportunity in an optimal way, it is necessary to find appropriate observables for deciphering the properties of dense and moderately hot matter in such collisions [2].

In recent theoretical studies of QCD at finite temperature and density, various inhomogeneous chiral condensed

\footnotetext{
*lee@ ruby.scphys.kyoto-u.ac.jp

e.nakano@s.kochi-u.ac.jp

tsue@kochi-u.ac.jp

\$tatsumi@ ruby.scphys.kyoto-u.ac.jp

b.friman@gsi.de
}

phases have been proposed (for a recent review see Ref. [3]). These studies suggest that the conventional QCD phase diagram should be redrawn. Indeed, it is possible that the phase structure at high net baryon densities and moderate temperatures is modified considerably by the presence of inhomogeneous phases. Thus, the region of the chiral transition may be extended and the order of the phase transition may change. These features are gleaned primarily from mean-field calculations in the Nambu-Jona-Lasinio (NJL) and quark-meson (QM) models [4,5], and the Dyson-Schwinger approach to dense QCD [6]. It is also interesting to note that within the Gor'kov approach to chiral effective models $[7,8]$ it is found that the QCD critical endpoint is a Lifshitz point, where the normal, homogeneous, and inhomogeneous chiral condensed phases meet. In the large $N_{c}$ approach to dense QCD, early studies suggested the emergence of the so-called chiral density wave $[9,10]$, while in the context of quarkyonic matter [11] another inhomogeneous phase, the so-called quarkyonic chiral spiral, was discussed [12].

The inhomogeneous chiral condensed phases mentioned above correspond to a one-dimensional modulation embedded in three spatial dimensions. Some of these structures are based on extrapolations from analytic solutions obtained for purely $(1+1)$-dimensional systems 
[13]. Possible extensions to higher-dimensional modulations have been studied, with the result that the onedimensional modulation tends to be favored close to the Lifshitz point [14] and/or at zero temperature [15].

Let us start by classifying the modulations for inhomogeneous phases, according to the convention employed in condensed matter physics. There are basically two types of one-dimensional modulations: one is of the Fulde-Ferrell (FF) type [16], characterized by modulations of the phase of a complex order parameter with constant amplitude, while the other is of the Larkin-Ovchinnikov (LO) type [17], where by contrast only the amplitude is modulated. The FF type includes the dual chiral density wave (DCDW) [4] and the quarkyonic chiral spiral [12]. On the other hand, the chiral density wave (a plane wave) $[9,10]$ and periodic domain walls [5] are of the LO type. In the present paper we focus on the DCDW, which is of FF type. The DCDW is characterized by modulated scalar and pseudoscalar condensates with a constant amplitude $\Delta$ and a wave number $q$ [4],

$$
\langle\bar{\psi} \psi\rangle=\Delta \cos q z, \quad\left\langle\bar{\psi} i \gamma_{5} \tau_{3} \psi\right\rangle=\Delta \sin q z
$$

where $\psi$ is the quark field for two flavors, and $\tau_{3}$ a Pauli matrix diagonal in the isospin space. This configuration is akin to $\sigma \pi^{0}$ condensation, obtained in neutron matter within the sigma model [18], and is thus expected to smoothly connect between nuclear and quark matter.

Most studies of inhomogeneous chiral condensed phases so far are based on mean-field calculations. Thus, effects of thermal and quantum fluctuations have yet to be studied. In the context of pion condensation, the stability of modulated condensates against thermal fluctuations has been studied on the basis of Landau-Peierls arguments [19]. It was found that in such systems there is no true long-range order with a nonvanishing order parameter [20]. Instead, such systems can develop a quasiordered one-dimensional condensate, with correlation functions that decay algebraically in space. ${ }^{1}$ In this paper, we investigate the stability of the DCDW phase against low-energy fluctuations of NambuGoldstone (NG) modes associated with the spontaneous symmetry breaking, along the lines of Ref. [20].

The paper is organized as follows. In the next section, we construct a $(3+1)$-dimensional Landau-Ginzburg-Wilson effective Lagrangian for general order parameters of the chiral condensate, which are allowed to be spacetime dependent, and then apply the formalism to the DCDW phase. In Sec. III, we discuss the symmetry breaking pattern and the corresponding NG modes in the DCDW phase. We also present the dispersion relations for these low-energy collective excitation modes, by introducing

\footnotetext{
${ }^{1}$ A similar state is found, e.g., in smectic liquid crystals $[21,22]$. See also Refs. [23,24] for a corresponding discussion on FFLO superconductors/superfluids.
}

fluctuations such as amplitudons and phonons/phasons (NG modes) on the ground state of the DCDW. In Sec. IV, we investigate the impact of low-energy fluctuations on the order parameter by evaluating the long-range correlation functions. Finally, Sec. V is devoted to summary and outlook.

\section{LANDAU-GINZBURG-WILSON EFFECTIVE LAGRANGIAN}

We start by introducing a $2 \times 2$ matrix field $M$ as the $(1 / 2,1 / 2)$ representation of the chiral $S U(2)_{L} \times S U(2)_{R}$ symmetry. In the following, we shall use the fact that $S U(2)_{L} \times S U(2)_{R}$ is isomorphic to the four-dimensional rotation group $O(4)$. The matrix $M$ can be expressed in terms of the right and left handed quark fields $\psi_{R, L}$ [25], $M=\bar{\psi}_{L} \psi_{R}$. Under the transformations $U_{R, L}=$ $\exp [-i(\vec{\alpha} \pm \vec{\beta}) \cdot \vec{\tau} / 2]$, where $\vec{\tau}$ is the isospin Pauli matrix and $\vec{\alpha}$ and $\vec{\beta}$ are two three-dimensional vector parameters, the matrix $M$ transforms (to leading order in $\vec{\alpha}$ and $\vec{\beta}$ ) as $M_{i j} \rightarrow M_{i j}+\frac{i}{2} \vec{\alpha} \cdot[\vec{\tau}, M]_{i j}+\frac{i}{2} \vec{\beta} \cdot\{\vec{\tau}, M\}_{i j}$. With the parametrization $M=\sigma+i \vec{\pi} \cdot \vec{\tau}$, one finds the corresponding transformation laws for $\sigma$ and $\vec{\pi}: \sigma \rightarrow \sigma-\vec{\beta} \cdot \vec{\pi}$ and $\vec{\pi} \rightarrow \vec{\pi}-\vec{\alpha} \times \vec{\pi}+\vec{\beta} \sigma$. Thus, the rotation with $\vec{\alpha}$ corresponds to the vector (isospin) rotation while that with $\vec{\beta}$ to an axial vector (chiral or axial isospin) rotation, respectively. We can then introduce a four-component composite field $\phi^{T}=(\sigma, \vec{\pi})$, which transforms as a four-dimensional vector under $O(4)$ rotations.

Now, we construct a low energy effective Lagrangian density $\mathcal{L}$ with $O(4)$ symmetry in terms of $\phi$ and its derivatives, ${ }^{2}$

$$
\begin{aligned}
\mathcal{L}= & c_{2} \partial_{0} \phi \cdot \partial_{0} \phi-\mathcal{V}, \\
\mathcal{V}= & a_{2} \phi \cdot \phi+a_{4,1}(\phi \cdot \phi)^{2}+a_{4,2} \boldsymbol{\nabla} \phi \cdot \nabla \phi \\
& +a_{6,1} \nabla^{2} \phi \cdot \nabla^{2} \phi+a_{6,2}(\nabla \phi \cdot \nabla \phi)(\phi \cdot \phi) \\
& +a_{6,3}(\phi \cdot \phi)^{3}+a_{6,4}(\phi \cdot \nabla \phi)^{2} .
\end{aligned}
$$

Note here that we have assumed that this Lagrangian is obtained from a microscopic theory, i.e., QCD, by integrating out all higher energy modes. Moreover, in-medium effects are by assumption implicitly subsumed in the coefficients, implying a loss of explicit Lorentz invariance. Thus, in the following, we deal only with the low energy modes, and study effects of low energy fluctuations on the inhomogeneous phase. The potential term $\mathcal{V}$ is expanded up to sixth order in powers of the field and fourth order in its

\footnotetext{
${ }^{2}$ For nonzero isospin charges, a term of the form $\frac{1}{2} \epsilon_{i j k l} \mu_{i j} Q_{i j}$ is added to the Lagrangian. Here $\epsilon_{i j k l}\left(\epsilon_{1234}=1\right)$ is the antisymmetric tensor, and $\mu_{i j}=-\mu_{j i}$ the chemical potential for the isospin density $Q_{i j}=i\left(\phi_{i} \partial_{0} \phi_{j}-\phi_{j} \partial_{0} \phi_{i}\right)$.
} 
derivatives, as required for stability of the inhomogeneous phase at the mean-field level.

Hereafter we set $c_{2}=1$ for simplicity. The expansion coefficients $a_{i, j}$ in $\mathcal{V}$ can be evaluated within effective chiral models, like the NJL [7] and QM [5,8] models. In the former, one finds the following relations among them $a_{4,1}=a_{4,2}$ and $\left(a_{6,1}, a_{6,2}, a_{6,4}\right)=(1 / 2,3,2) a_{6,3}$.

\section{LOW ENERGY EFFECTIVE MODES IN DCDW PHASE}

We consider an inhomogeneous time independent chiral condensate of the DCDW type,

$$
\phi_{0}^{T}=\Delta(\cos q z, 0,0, \sin q z),
$$

where $\Delta$ is a constant amplitude corresponding to $\left\langle\bar{\psi} e^{i \gamma_{5} \tau_{3} \mathbf{q} \cdot \mathbf{r}} \psi\right\rangle$ and $q$ is the wave number of modulation in the $z$ direction. The values of $\Delta$ and $q$ are determined by minimizing the potential term of the Lagrangian. For the condensate $\phi_{0}$, the potential term reads

$$
\begin{aligned}
\mathcal{V}\left(\phi_{0}\right)= & a_{2} \Delta^{2}+a_{4,1} \Delta^{4}+a_{4,2} q^{2} \Delta^{2} \\
& +a_{6,1} q^{4} \Delta^{2}+a_{6,2} q^{2} \Delta^{4}+a_{6,3} \Delta^{6} .
\end{aligned}
$$

Stability of the inhomogeneous phase is guaranteed by

$a_{6,1}>0\left(\right.$ or $\left.\quad a_{6,3}>0\right)$, and $a_{6,1} a_{6,3}-a_{6,2}^{2} / 4>0$.

The stationary conditions for $q$ and $\Delta, \frac{\partial \mathcal{V}}{\partial \Delta}=\frac{\partial \mathcal{V}}{\partial q}=0$, yield

$$
\begin{aligned}
& 2 q \Delta^{2}\left(a_{4,2}+2 a_{6,1} q^{2}+a_{6,2} \Delta^{2}\right)=0, \\
& 2 \Delta\left[a_{2}+a_{4,2} q^{2}+a_{6,1} q^{4}\right. \\
& \left.\quad+2\left(a_{4,1}+a_{6,2} q^{2}\right) \Delta^{2}+3 a_{6,3} \Delta^{4}\right]=0 .
\end{aligned}
$$

They admit three types of solutions:

(i) Normal phase: $\Delta=0$,

(ii) Homogeneous chiral condensed phase: $q=0$, $\Delta \neq 0$,

(iii) Inhomogeneous chiral condensed phase: $q^{2}=$ $-\left(a_{4,2}+a_{6,2} \Delta^{2}\right) / 2 a_{6,1}, \Delta \neq 0$.

For a given set of coefficients, the phase with the lowest energy is realized on the classical level. The coefficients implicitly depend on the medium, and are thus functions of thermodynamic variables, like temperature and chemical potentials. Throughout the paper we do not fix the coefficients, assuming the DCDW phase is realized for a given set of coefficients. The general feature of phase boundary is discussed in Appendix A.

\section{A. Symmetry breaking and Nambu-Goldstone modes in DCDW phase}

In the DCDW phase with nonvanishing $\Delta$ and $q$, the $S U(2)_{L} \times S U(2)_{R}$ chiral symmetry, as well as the translational invariance in the $z$ direction and the symmetry under rotations about the $x$ and $y$ axes are spontaneously broken. To see the symmetry breaking pattern explicitly, we first perform infinitesimal transformations corresponding to a spatial translation in the $z$ direction with a displacement parameter $s$ and a chiral rotation through the angles $\vec{\alpha}$ and $\vec{\beta}$ :

$$
\phi_{0} \rightarrow \phi_{0}+\Delta\left(\begin{array}{c}
-\left(s q+\beta_{3}\right) \sin q z \\
\beta_{1} \cos q z-\alpha_{2} \sin q z \\
\beta_{2} \cos q z+\alpha_{1} \sin q z \\
\left(s q+\beta_{3}\right) \cos q z
\end{array}\right)
$$

The form of the first and the fourth components implies that $\phi_{0}$ is invariant under a simultaneous spatial translation and axial isospin rotation about the $z$ axis through the angle $\beta_{3}$, if $q s+\beta_{3}=0$. Thus, in the DCDW phase a locking of axial isospin rotations with translations is realized. In terms of symmetry generators, there are two unique orthogonal linear combinations of $s$ and $\beta_{3}$; one corresponding to a broken generator, the other to an unbroken one. Consequently, the corresponding NG mode is generated by a transformation with $q s+$ $\beta_{3} \neq 0$, i.e., by one whose generator is broken in the DCDW phase. In the following, we use $\beta_{3}=\beta_{3}(t, \vec{x})$ and $s=0$ to generate the NG mode associated with the broken generator. Similar arguments for the spontaneous breakdown of internal and spacetime symmetries are given in Refs. [26,27].

The rotations through $\beta_{1}$ and $\alpha_{2}$ generate variations in the second component in Eq. (9). However, the corresponding NG modes are linearly dependent in the sense discussed in Ref. [28]. In case of a vanishing wave number $q=0$, only $\beta_{1}$ is relevant. Thus, we generate the corresponding $\mathrm{NG}$ mode using $\beta_{1}=$ $\beta_{1}(t, \vec{x})$ and $\alpha_{2}=0$. Analogous arguments can be applied the third component in Eq. (9), thus eliminating $\alpha_{1}$ in favor of $\beta_{2}$.

Spatial rotations about the $x$-axis by an angle $\theta_{x}$ yields a transformation, which is nonuniform in space: $z \rightarrow z \cos \theta_{x}+y \sin \theta_{x}$. Similarly, the rotations about the $y$-axis by $\theta_{y}$ yields the analogous transformation. However, the corresponding NG modes and those generated by translations are also linearly dependent [28].

We conclude that, although there are eight broken generators for internal and space time symmetries in the DCDW phase, only three independent NG modes remain. These can be chosen as the axial isospin rotations generated by $\vec{\beta}=\vec{\beta}(t, \vec{x})$. 
LEE et al.

PHYSICAL REVIEW D 92, 034024 (2015)

\section{B. Low energy collective excitations}

We now consider a general fluctuation in the DCDW phase:

$$
\begin{aligned}
\phi & =(\Delta+\delta)\left(\begin{array}{l}
\cos \left(q z+\beta_{3}\right) \cos \beta_{2} \cos \beta_{1} \\
\cos \left(q z+\beta_{3}\right) \cos \beta_{2} \sin \beta_{1} \\
\cos \left(q z+\beta_{3}\right) \sin \beta_{2} \\
\sin \left(q z+\beta_{3}\right)
\end{array}\right) \\
& =(\Delta+\delta) U\left(\beta_{i}\right)\left(\begin{array}{c}
\cos (q z) \\
0 \\
0 \\
\sin (q z)
\end{array}\right) .
\end{aligned}
$$

Here $\delta$ is the amplitude fluctuation, the parameters $\vec{\beta}=$ $\left\{\beta_{1}, \beta_{2}, \beta_{3}\right\}$ specifies a rotation in the four-dimensional space spanned by the $\sigma$ and $\vec{\pi}$ fields. Finally, $U\left(\beta_{i=1,2,3}\right):=$ $e^{i \beta_{1} L_{1}} e^{i \beta_{2} L_{2}} e^{i \beta_{3} L_{3}}$, where $L_{1,2,3}$ are the $\mathrm{O}(4)$ (axial isospin) generators [25]. This parametrization clearly shows that the displacement in the $z$ direction is equivalent to a chiral rotation through $\beta_{3}$. To leading order in the fluctuations, Eq. (10) yields

$\phi=(1+\delta) \phi_{0}+\Delta\left(\begin{array}{c}-\beta_{3} \sin q z \\ \beta_{1} \cos q z \\ \beta_{2} \cos q z \\ \beta_{3} \cos q z\end{array}\right)+\mathcal{O}\left(\beta_{i}^{2}, \delta \beta_{i}, \delta^{2}\right)$,

which exhibits the fluctuation of the amplitude in addition to the fluctuations corresponding to the NG modes. In the following we consider local fluctuations, promoting the parameters $\delta$ and $\vec{\beta}$ to fields $\delta(x)$ and $\vec{\beta}(x)$, where we use the compact notation $x \equiv\{t, \vec{x}\}$.
Plugging the above parametrization into the Lagrangian, we can systematically derive a low energy effective theory by expanding in powers of the fluctuation fields $\delta$ and $\vec{\beta}$. Up to the second order in the fields, the Lagrangian $L=$ $\int \mathrm{d}^{3} x \mathcal{L}$ reads

$$
\begin{aligned}
\mathcal{L}= & \left(\partial_{0} \delta\right)^{2}+\Delta^{2}\left(\partial_{0} \vec{\beta}_{U}\right)^{2}+\Delta^{2}\left(\partial_{0} \beta_{3}\right)^{2} \\
& -\left(\mathcal{V}_{\delta}+\mathcal{V}_{\delta \beta}+\mathcal{V}_{\beta}\right),
\end{aligned}
$$

where

$$
\begin{aligned}
\mathcal{V}_{\delta}= & M^{2} \delta^{2}+a_{6,4} \Delta^{2}(\nabla \delta)^{2} \\
& +4 a_{6,1} q^{2}\left(\nabla_{z} \delta\right)^{2}+a_{6,1}\left(\nabla^{2} \delta\right)^{2}, \\
\mathcal{V}_{\delta \beta}= & 4 q \Delta\left[a_{6,2} \Delta^{2} \delta-2 a_{6,1} \nabla^{2} \delta\right] \nabla_{z} \beta_{3}, \\
\mathcal{V}_{\beta}= & a_{6,1} \Delta^{2}\left(\nabla^{2} \vec{\beta}_{U}+q^{2} \vec{\beta}_{U}\right)^{2} \\
& +a_{6,1} \Delta^{2}\left[\left(\nabla^{2} \beta_{3}\right)^{2}+4 q^{2}\left(\nabla_{z} \beta_{3}\right)^{2}\right],
\end{aligned}
$$

with the mass term $M^{2}=4\left(a_{4,1}+a_{6,2} q^{2}\right) \Delta^{2}+12 a_{6,3} \Delta^{4}$ and the transverse field $\vec{\beta}_{U} \equiv \vec{\beta}_{T} \cos q z$ where $\vec{\beta}_{T}=$ $\left\{\beta_{1}, \beta_{2}\right\}$. In the above equations the stationary condition $a_{4,2}+a_{6,2} \Delta^{2}+2 q^{2} a_{6,1}=0$ has been used. For details of the derivation, see Appendix B. Here we are interested in the low energy NG modes in the DCDW phase. To this end, the Lagrangian given above is sufficient.

In order to investigate the thermodynamics of the system, we now move to Euclidean space: $t \rightarrow-i \tau$ with the period $0 \leq \tau \leq \beta$ where $\beta=1 / T$ is the inverse temperature. For Gaussian fluctuations, we obtain the Euclidean action in Fourier space (for details we refer the reader to Appendix C),

$$
\begin{aligned}
-S_{E}=\int_{0}^{\beta} \mathrm{d} \tau \int \mathrm{d}^{3} x \mathcal{L}_{E}= & \mathcal{S} \mathrm{d} k\left(\begin{array}{c}
\delta^{*}(k) \\
\Delta \beta_{3}^{*}(k)
\end{array}\right)^{T}\left(\begin{array}{cc}
S_{\delta 0}^{-1}(k) & -g(k) \\
g(k) & S_{0}^{-1}(k)
\end{array}\right)\left(\begin{array}{c}
\delta(k) \\
\Delta \beta_{3}(k)
\end{array}\right) \\
& +\frac{1}{4} \oint \mathrm{d} k\left(\begin{array}{c}
\Delta \vec{\beta}_{T}^{*}(k) \\
\Delta \vec{\beta}_{T}^{*}(k+2 q \hat{z})
\end{array}\right)^{T}\left(\begin{array}{cc}
S_{0}^{-1}(k) & G(k) \\
G(k) & S_{0}^{-1}(k+2 q \hat{z})
\end{array}\right)\left(\begin{array}{c}
\Delta \vec{\beta}_{T}(k) \\
\Delta \vec{\beta}_{T}(k+2 q \hat{z})
\end{array}\right),
\end{aligned}
$$

where we have used the shorthand notation: $£ \mathrm{~d} k \equiv T \sum_{n} \int \frac{\mathrm{d}^{3} k}{(2 \pi)^{3}}$, and $k=\left(\omega_{n}, \vec{k}\right)$ with the Matsubara frequency $\omega_{n}=2 \pi n T(\equiv i \omega)$. The inverse propagators in the above matrix notation are given by

$$
\begin{aligned}
S_{\delta 0}^{-1}(k) & =\omega^{2}-\left[M^{2}+a_{6,4} \Delta^{2} \vec{k}^{2}+4 a_{6,1} q^{2}\left(k_{z}\right)^{2}+a_{6,1}\left(\vec{k}^{2}\right)^{2}\right], \\
g(k) & =2 i q\left[a_{6,2} \Delta^{2}+2 a_{6,1} \vec{k}^{2}\right] k_{z}, \\
S_{0}^{-1}(k) & =\omega^{2}-a_{6,1}\left[4 q^{2} k_{z}^{2}+\left(\vec{k}^{2}\right)^{2}\right], \\
\text { and } \quad G(k) & =\omega^{2}-a_{6,1}\left(\vec{k}^{2}+2 q k_{z}\right)^{2} .
\end{aligned}
$$

We note that for a nonvanishing wave number $q$, the $\delta$ and $\beta_{3}$ fluctuations mix. Moreover, transverse fluctuations $\vec{\beta}_{T}$ with different momenta $k$ and $k+2 q \hat{z}$ mix, owing to the scattering of fluctuations off the background modulation. 
The determinant of the first matrix, $S_{\delta 0}^{-1}(k) S_{0}^{-1}(k)+$ $g^{2}(k)=0$, yields the dispersion relations of the normal modes involving $\delta$ and $\beta_{3}$,

$$
\begin{gathered}
\omega_{+}^{2} \simeq M^{2}+a_{6,1}\left[u_{z+}^{2} k_{z}^{2}+\left(\vec{k}^{2}\right)^{2}\right] \\
+a_{6,4} \Delta^{2} \vec{k}^{2}+A \vec{k}^{2} k_{z}^{2}+B k_{z}^{4}, \\
\omega_{-}^{2} \simeq a_{6,1}\left[u_{z-}^{2} k_{z}^{2}+\left(\vec{k}^{2}\right)^{2}\right]-A \vec{k}^{2} k_{z}^{2}-B k_{z}^{4},
\end{gathered}
$$

where $\quad u_{z \pm}^{2}=4 q^{2}\left(1 \pm \frac{a_{6.2}^{2} \Delta^{4}}{a_{6.1} M^{2}}\right), \quad A \equiv 4 q^{2} \Delta^{2} a_{6.2}\left(4 M^{2} a_{6.1}-\right.$ $\left.\Delta^{4} a_{6.2} a_{6.4}\right) / M^{4}$, and $B \equiv-\left(2 q \Delta^{2} a_{6.2}\right)^{4} / M^{6}$. Note that in the massless mode $\omega_{-}$, the sign of $u_{z-}^{2}$ is always positive in the inhomogeneous phase, and the dependence on the transverse momentum is subleading, $\mathcal{O}\left(k^{4}\right)$. Consequently, the transverse fluctuations are softer than the longitudinal ones.

Similarly, equating the determinant of the second matrix to zero, $S_{0}^{-1}(k) S_{0}^{-1}(k)-G^{2}(k)=0$, we obtain the dispersion relation for $\vec{\beta}_{T}$,

$\omega_{k}^{2}=a_{6,1}\left[4 q^{2} k_{z}^{2}+\left(\vec{k}^{2}\right)^{2}\right]-a_{6,1} \frac{2 k_{z}^{2}\left(\vec{k}^{2}\right)^{2}}{4 q^{2}+6 q k_{z}+2 k_{z}^{2}+\vec{k}^{2}}$.

The second term with the negative sign is a higher order correction of $\mathcal{O}\left(k^{6}\right)$, stemming from interactions with the background modulation. This term is irrelevant for the effects of low energy fluctuations and is therefore dropped in the following discussion.

\section{IMPACTS OF LOW ENERGY FLUCTUATIONS}

At low temperatures, the low energy fluctuations about the classical DCDW state dominate. We evaluate the contribution of Gaussian fluctuations to the partition function

$$
Z=\int[\mathcal{D} \delta][\mathcal{D} \Delta \vec{\beta}] e^{-S_{E}}
$$

Higher-order derivative corrections are dropped, with the assumption that fluctuations at energies above some cutoff $\Lambda$ have been integrated out in the effective Lagrangian (2), which then involves only the low-energy fluctuations, $\delta$ and $\vec{\beta}$, explicitly.

We first explore the impact of low energy fluctuations on the order parameter,

$$
\left\langle(\Delta+\delta) U\left(\beta_{i}\right) \phi_{0}\right\rangle=\Delta\left\langle U\left(\beta_{i}\right) \phi_{0}\right\rangle+\left\langle\delta U\left(\beta_{i}\right) \phi_{0}\right\rangle,
$$

where we use the compact notation $\langle\cdots\rangle \equiv$ $\int[\mathcal{D} \delta][\mathcal{D} \Delta \vec{\beta}] \cdots e^{-S_{E}} / Z$. In the Gaussian approximation, the two contributions to the expectation value reduce to

$$
\left\langle U\left(\beta_{i}\right) \phi_{0}\right\rangle \simeq\left(\begin{array}{l}
\cos (q z) e^{-\sum_{i}\left\langle\beta_{i}^{2}\right\rangle / 2} \\
0 \\
0 \\
\sin (q z) e^{-\left\langle\beta_{3}^{2}\right\rangle / 2}
\end{array}\right)
$$

and

$$
\left\langle\delta U\left(\beta_{i}\right) \phi_{0}\right\rangle \simeq\left(\begin{array}{l}
-\sin (q z)\left\langle\delta \beta_{3}\right\rangle e^{-\sum_{i}\left\langle\beta_{i}^{2}\right\rangle / 2} \\
0 \\
0 \\
\cos (q z)\left\langle\delta \beta_{3}\right\rangle e^{-\left\langle\beta_{3}^{2}\right\rangle / 2}
\end{array}\right) .
$$

Here the second order fluctuations are given by

$$
\begin{gathered}
\left\langle\delta(x) \beta_{3}(x)\right\rangle \simeq 0, \\
\Delta^{2}\left\langle\beta_{1,2}^{2}(x)\right\rangle \simeq \frac{1}{2} \int \frac{\mathrm{d}^{3} k}{(2 \pi)^{3}} \frac{T}{\omega_{k}^{2}}, \\
\text { and } \quad \Delta^{2}\left\langle\beta_{3}^{2}(x)\right\rangle \simeq \frac{1}{2} \int \frac{\mathrm{d}^{3} k}{(2 \pi)^{3}} \frac{T}{\omega_{-}^{2}},
\end{gathered}
$$

where the fluctuations $\left\langle\beta_{1,2,3}^{2}(x)\right\rangle$ are all logarithmically divergent due to the soft modes in the transverse directions. Details of the derivation are given in Appendix D. Consequently, the low-energy fluctuations wash out the order parameter, i.e., they destroy the off-diagonal longrange order,

$$
\left\langle(\Delta+\delta) U\left(\beta_{i}\right) \phi_{0}\right\rangle=0 .
$$

This result implies that a DCDW phase with true longrange order strictly speaking does not exist at nonzero temperature. Such a phase may, however, be realized in a modified form, with a quasi-long-range order (QLRO), analogous to that in the Berezinsky-Kosterlitz-Thouless phase in two-dimensional systems [29] and in smectic liquid crystals [22]. As we discuss in the next section, the quasi-long-range order is characterized by a power-law decay of the order parameter correlation function.

At zero temperature, on the other hand, quantum fluctuations are not strong enough to break the modulating order. In fact, at $T=0$ the second order fluctuations are given by the infrared convergent integral $\Delta^{2}\left\langle\beta_{1,2(3)}^{2}(x)\right\rangle \simeq$ $\frac{1}{4} \int \frac{\mathrm{d}^{3} k}{(2 \pi)^{3}} \frac{1}{\omega_{k(-)}}$, obtained by taking the zero temperature limit of Eqs. (D6) and (D12) in Appendix D.

The results of this section imply that within the Gaussian approximation the transition temperature of the true DCDW phase is $T_{\mathrm{DCDW}}=0$, and the region of $T>0$ is critical (QLRO). Now assume that there is a critical temperature $T_{c}>0$, where the system becomes unstable with respect to the formation of a state with a modulated 
order parameter. Then a quasi-one-dimensionally ordered phase or a phase with true long-range order in two- or threedimensional modulations may be realized below $T_{c}$ [20]. To determine which phase is preferred, one must, in principle, compare their free energies. Considering the different nature of these phases, this is a challenging task.

\section{A. Long-range correlations}

We now explore the behavior of the correlation functions in the Gaussian approximation. Since the order parameter is vectorlike, we define correlation functions among the components:

$$
f_{i j}(x)=\left\langle\phi_{i}(x) \phi_{j}^{*}(0)\right\rangle .
$$

These correlation functions are spatially anisotropic owing to the one-dimensional modulation of the background. We compute the dependence of the isoscalar correlation function on $z$. The diagonal components which contribute to the scalar channel are of the form:

$$
f_{11}(\hat{z} z) \simeq \frac{1}{8} \Delta^{2} \cos q z e^{-\sum_{i=1,2,3}\left\langle\left(\beta_{i}^{-}\right)^{2}\right\rangle / 2},
$$

where $\beta_{i}^{-} \equiv \beta_{i}(z)-\beta_{i}(0)$. For details we refer to Appendix E.

Similar results are obtained for the other components:

$$
\begin{aligned}
& f_{22}(\hat{z} z) \simeq \frac{1}{8} \Delta^{2} \cos q z e^{-\sum_{i=1,2,3}\left\langle\left(\beta_{i}^{-}\right)^{2}\right\rangle / 2}, \\
& f_{33}(\hat{z} z) \simeq \frac{1}{4} \Delta^{2} \cos q z e^{-\sum_{i=2,3}\left\langle\left(\beta_{i}^{-}\right)^{2}\right\rangle / 2}, \\
& \text { and } f_{44}(\hat{z} z) \simeq \frac{1}{2} \Delta^{2} \cos q z e^{-\left\langle\left(\beta_{3}^{-}\right)^{2}\right\rangle / 2} .
\end{aligned}
$$

Here the exponents, $\left\langle\left(\beta_{i}^{-}\right)^{2}\right\rangle$, exhibit the following functional form at large $z$,

$$
\begin{aligned}
\left\langle\left(\beta_{1,2(3)}^{-}\right)^{2}\right\rangle / 2 & \simeq \frac{T}{2 \Delta^{2}} \int \frac{\mathrm{d}^{3} k}{(2 \pi)^{3}} \frac{1-\cos k_{z} z}{\omega_{k(-)}^{2}} \\
& \simeq \frac{T}{16 \pi a_{6,1} \Delta^{2} u_{z-}} \ln \frac{z \Lambda^{2}}{2 q}
\end{aligned}
$$

where $\Lambda$ is an ultraviolet cutoff.

Putting it all together, we obtain the long-range scalar correlation in the $z$ direction,

$$
\left\langle\phi(z \hat{z}) \cdot \phi^{*}(0)\right\rangle \sim \frac{1}{2} \Delta^{2} \cos q z\left(\frac{z}{z_{0}}\right)^{-T / T_{0}}
$$

where $z_{0} \equiv 2 q / \Lambda^{2}$, and $T_{0} \equiv 16 \pi a_{6,1} \Delta^{2} u_{z_{-}}$. In a similar manner, we compute the form of the long-range correlation function in transverse directions,

$$
\left\langle\phi\left(x_{t} \hat{x}_{t}\right) \cdot \phi^{*}(0)\right\rangle \sim \frac{1}{2} \Delta^{2}\left(\frac{x_{t}}{x_{0}}\right)^{-2 T / T_{0}},
$$

where $x_{0} \equiv \Lambda^{-1}, x_{t}$ is the transverse distance, and the factor 2 in the exponent of $x_{t} / x_{0}$ reflects the number of transverse directions. Note that, in contrast to the longitudinal direction, there is no modulation of the correlation function in the transverse directions.

In this section we have shown that quasi-long-range order of the one-dimensional DCDW phase features algebraically decaying correlation functions at large distances. The slow decay of the spatial correlations distinguish the quasiordered phase from normal or disordered phases, characterized by exponential decays. Depending on the experimental resolution and finite size effects, the algebraic correlations can effectively mimic true long-range order [20,21,29].

\section{SUMMARY AND OUTLOOK}

In this paper we have explored the soft modes of an inhomogeneous chiral condensed phase with onedimensional modulation, the DCDW phase. We found that this phase exhibits a flavor-translation locking symmetry and clarified the counting of Nambu-Goldstone modes. The dispersion relations for collective excitations, including the NG modes, were derived. The low-energy modes are spatially anisotropic and particularly soft in the directions transverse to the modulation, owing to the lack of terms quadratic in the transverse momentum in the dispersion relations. As in smectic liquid crystals, the absence of such terms is a consequence of the symmetry under rotations about any axis orthogonal to the modulation direction [19,22].

Moreover, we have shown that at nonzero temperatures the DCDW phase exhibits a Landau-Peierls instability, i.e., the long range order is destroyed by low-energy (long-wavelength) fluctuations of the order parameter. Nevertheless, a phase similar to the smectic phases of liquid crystals, characterized by a quasi-long-range order with algebraically decaying order parameter correlation functions, is possible. Such an "algebraic order" can, depending on the conditions, emulate true long-range order. In particular, this would be the case, in a finite systems, where the range of the order-parameter correlations exceeds the size of the system.

The experimental verification of "algebraic order" can be challenging. The slow decay of the correlations has been observed by light scattering in smectic-A liquid crystals [21], by neutron scattering in Bragg glass [30] and only recently in a two-dimensional system of the Berezinsky-Kosterlitz-Thouless type [31], by measuring the coherence of photons emitted in quasiparticle decays. Whether the quasi-one-dimensionally ordered DCDW phase could be observed by an appropriate choice of probes is still an open question. Hence, it 
would be important to systematically explore how the collective modes in the DCDW phase interact with external probes such as hadrons (quarks) and photons (gauge fields).

There are also several theoretical issues, that deserve further study. In particular, it is known that inhomogeneous chiral phases are favored in systems with vector-vector type interactions, which tend to enhance the size of the inhomogeneous area [32], and that in the presence of an external magnetic field the FF type phase is stabilized, also at finite temperatures, by topological aspects $[33,34]$. Moreover, since two- and threedimensional condensates with true long-range order are allowed at any temperature [19], it would be important to compare the free energy of such phases with that of a one-dimensional condensate. It would also be interesting to understand how higher order interactions among the collective modes modify the soft modes. These may affect the Landau-Peierls instability of inhomogeneous phases discussed here.

Finally, the topics discussed here may have an impact on the physics of compact stars. It has been speculated that various spatially inhomogeneous phases, like nuclear pasta phases [35] and hadron-quark mixed phases [36], could be realized in the interior of such stars. These could have phenomenological implications, allowing, e.g., novel cooling scenarios [37]. Thus, it would be interesting to study the properties of inhomogeneous chiral condensed phases under conditions relevant for neutron stars in general and compact stars with quark cores in particular [38], i.e., in charge neutral matter in $\beta$ equilibrium but also at nonzero isospin density [39] and finite strangeness [40], and also to see how the NG modes in such phases affect transport properties in the inner core of compact stars. These topics give interesting directions for future works.

\section{ACKNOWLEDGMENTS}

T.-G. L. would like to thank Ryo Yoshiike, Toshiki Maruyama, Kei Iida, and Kazuhiko Kamikado for useful comments and discussions. This work is partially supported by Grant-in-Aid for Scientific Research on Innovative Areas through No. 24105008 provided by MEXT.

\section{APPENDIX A: DISCUSSION ON PHASE BOUNDARY}

We give a general phase structure for the DCDW type inhomogeneous phase in the coefficient space. Here we define $\mathcal{V}\left(\Delta^{2}, q^{2}\right) \equiv \mathcal{V}\left(\phi_{0}\right)$ for the potential Eq. (5). The potential at the stationary point with respect to $q,\left.\frac{\partial \mathcal{V}}{\partial q^{2}}\right|_{q=q_{0}}=0$ where $q_{0}^{2}=-\left(a_{4.2}+a_{6.2} \Delta^{2}\right) / 2 a_{6.1}$, is given by

$$
\begin{aligned}
\mathcal{V}\left(\Delta^{2}, q_{0}^{2}\right)= & \left(a_{2}-\frac{a_{4.2}^{2}}{4 a_{6.1}}\right) \Delta^{2}+\left(a_{4.1}-\frac{a_{4.2} a_{6.2}}{2 a_{6.1}}\right) \Delta^{4} \\
& +\left(a_{6.3}-\frac{a_{6.2}^{2}}{4 a_{6.1}}\right) \Delta^{6} \\
\equiv & A \Delta^{2}+B \Delta^{4}+C \Delta^{6} .
\end{aligned}
$$

$\mathcal{V}\left(\Delta^{2}, q_{0}^{2}\right)$ has stationary points with respect to $\Delta$ at $\Delta^{2}=0$, $\Delta_{-}^{2}$, and $\Delta_{+}^{2}$, where

$$
\Delta_{ \pm}^{2}=\frac{-B \pm \sqrt{B^{2}-3 A C}}{3 C}
$$

and $\Delta_{+}^{2}$ corresponds to the nontrivial phase.

To determine the phase boundaries we define the difference of the potential energies between $q=0$ and $q \neq 0$ by

$$
\begin{aligned}
\mathcal{V}_{d}\left(\Delta^{2}, q^{2}\right) & \equiv \mathcal{V}\left(\Delta^{2}, q^{2}\right)-\mathcal{V}\left(\Delta^{2}, 0\right) \\
& =q^{2} \Delta^{2}\left(a_{4.2}+a_{6.1} q^{2}+a_{6.2} \Delta^{2}\right) .
\end{aligned}
$$

At the stationary point where $q=q_{0}$, it becomes negative semidefinite

$$
\begin{aligned}
\mathcal{V}_{d}\left(\Delta^{2}, q_{0}^{2}\right) & =q_{0}^{2} \Delta^{2}\left(a_{4.2}+a_{6.1} q_{0}^{2}+a_{6.2} \Delta^{2}\right) \\
& =-a_{6.1} q_{0}^{4} \Delta^{2} \leq 0,
\end{aligned}
$$

because $a_{6.1}>0$ for the stability. The above result shows that possible phase transitions from $(q \neq 0$, $\Delta \neq 0)$ to $(q=0, \Delta \neq 0)$ take place at $\mathcal{V}_{d}\left(\Delta_{0}^{2}, q_{0}^{2}\right)=0$ and $\mathcal{V}(0,0)>\mathcal{V}\left(\Delta_{0}^{2}, 0\right)$, where $\Delta_{0}$ satisfies the stationary condition $\left.\frac{\partial \mathcal{V}\left(\Delta^{2}, 0\right)}{\partial \Delta^{2}}\right|_{\Delta=\Delta_{0}}=\left.\frac{\partial \mathcal{V}_{d}\left(\Delta_{0}^{2}, q_{0}^{2}\right)}{\partial \Delta^{2}}\right|_{\Delta=\Delta_{0}}=0 \quad$ only at $\left(q_{0}=0, \Delta_{0}^{2}=-a_{4.2} / a_{6.2}\right)$. This gives the transition condition for $a_{4.2}<0$ and $a_{6.1} a_{6.3}>a_{6.2}^{2} / 4$ :

$$
\frac{-a_{4.1}+\sqrt{a_{4.1}^{2}-3 a_{2} a_{6.1}}}{3 a_{6.1}}=-\frac{a_{4.2}}{a_{6.2}} .
$$

Therefore, at the phase boundaries between inhomogeneous to homogeneous chiral condensed phases, $q$ continuously drops to zero, and $\Delta$ also changes continuously but may not smoothly, because the potential minimum switches from $\mathcal{V}\left(\Delta^{2}, 0\right)$ to $\mathcal{V}\left(\Delta^{2}, q_{0}^{2}\right)$. In this case the potential shape for $\Delta$ is of the first or the second order type, depending on $a_{2}>0, a_{4.1}<0$ or $a_{2}<0, a_{4.1}>0$, respectively.

On the other hand, the possible transitions from $(q \neq 0, \Delta \neq 0)$ to $(\Delta=0)$ may be of the first (discontinuous) or the second order (continuous) for both $q$ and $\Delta$. In this case the above argument is also applicable; only difference is that at the phase boundaries $\mathcal{V}\left(\Delta_{0}^{2}, 0\right)>$ $\mathcal{V}\left(\Delta_{0}^{2}, q_{0}^{2}\right)=\mathcal{V}(0,0)$. 
Note here that the phase structures obtained above do not describe a first order transition from the DCDW phase to the hadronic phase obtained in the NJL model in the mean-field approximation [4], where $q$ becomes discontinuous. It implies that we need higher order derivative terms of $\mathcal{O}\left(\nabla^{6}\right)$, and consequently need terms of eighth order of the mass dimension for the description of the first order transition from $(q \neq 0, \Delta \neq 0)$ to $(q=0, \Delta \neq 0)$. While, the DCDW phase obtained from Dyson-Schwinger type approximation to QCD [6] has boundaries of the first and the second order. The order of phase transitions depends on microscopic models and approximations. Since our main purpose is to investigate the stability of the inhomogeneous chiral condensed phase against the low energy fluctuations, the inclusion of higher derivative terms does not change conclusion qualitatively. Also, we do not discuss the fluctuation effects on the possible modification of the effective potential.

\section{APPENDIX B: EFFECTIVE ACTION OF FLUCTUATIONS}

We derive the effective action of the fluctuations to the second order of field expansion. It mostly comes from derivative terms: $(\nabla \phi)^{2}$ and $\left(\nabla^{2} \phi\right)^{2}$. First of all, we rewrite the field as

$$
\phi(x)=(\Delta+\delta) U\left(\beta_{i}\right) \phi_{0}(x)=(\Delta+\delta) V\left(\beta_{i}\right) \hat{\phi}_{0},
$$

where $V \equiv U S$ with $S=e^{q z L_{3}}$, and $\hat{\phi}_{0}=(1,0,0,0)^{T}$.

\section{1. $(\nabla \phi)^{2}$ term}

We derive the derivative term $(\nabla \phi)^{2}$ up to the second order of fluctuation fields:

$$
\begin{aligned}
\nabla \phi \cdot \nabla \phi= & \hat{\phi}_{0}^{T}\left[\nabla \delta V^{-1}+(\Delta+\delta) \nabla V^{-1}\right] \\
& \times[\nabla \delta V+(\Delta+\delta) \nabla V] \hat{\phi}_{0} \\
\simeq & (\nabla \delta)^{2}+q^{2} \delta^{2}+2 \Delta \delta\left(q^{2}+2 q \nabla_{z} \beta_{3}\right),
\end{aligned}
$$

where $\hat{\phi}_{0}^{T}\left[\nabla V^{-1} \nabla V\right] \hat{\phi}_{0}=q^{2}+2 q \nabla_{z} \beta_{3}+q^{2} \beta_{3}^{2}+\left(\nabla \beta_{3}\right)^{2}$, and we have used the stationary condition under which $a_{4.2}, a_{6.1}$, and $a_{6.2}$ terms disappear.

\section{2. $\left(\nabla^{2} \phi\right)^{2}$ term}

We next derive the derivative term $\left(\nabla^{2} \phi\right)^{2}$ :

$$
\begin{aligned}
\nabla^{2} \phi \cdot \nabla^{2} \phi= & \hat{\phi}_{0}^{T}\left[\nabla^{2}\left(\delta V^{-1}\right)+\Delta \nabla^{2} V^{-1}\right] \\
& \times\left[\nabla^{2}(\delta V)+\Delta \nabla^{2} V\right] \hat{\phi}_{0} \\
= & \left\langle\nabla^{2}\left(\delta V^{-1}\right) \nabla^{2}(\delta V)\right\rangle+\Delta\left\langle\nabla^{2}\left(\delta V^{-1}\right) \nabla^{2} V\right\rangle \\
& +\Delta\left\langle\nabla^{2} V^{-1} \nabla^{2}(\delta V)\right\rangle+\Delta^{2}\left\langle\nabla^{2} V^{-1} \nabla^{2} V\right\rangle,
\end{aligned}
$$

where $\langle\cdots\rangle \equiv \hat{\phi}_{0}^{T} \cdots \hat{\phi}_{0}$. We expand each term up to second order of the fluctuation fields. Hereafter we chop off terms which will disappear together with $a_{4.2}, a_{6.1}$, and $a_{6.2}$ terms under the stationary condition, and the constants. Omitting total derivatives, we obtain

$$
\begin{gathered}
\left\langle\nabla^{2}\left(\delta V^{-1}\right) \nabla^{2}(\delta V)\right\rangle \simeq\left(\nabla^{2} \delta-q^{2} \delta\right)^{2}+4 q^{2}\left(\nabla_{z} \delta\right)^{2}, \\
\left\langle\nabla^{2}\left(\delta V^{-1}\right) \nabla^{2} V\right\rangle \simeq q^{4} \delta+4 q \nabla^{2} \nabla_{z} \delta \beta_{3}-4 q^{3} \nabla_{z} \delta \beta_{3}, \\
\left\langle\nabla^{2} V^{-1} \nabla^{2}(\delta V)\right\rangle=\left\langle\nabla^{2}\left(\delta V^{-1}\right) \nabla^{2} V\right\rangle, \\
\left\langle\nabla^{2} V^{-1} \nabla^{2} V\right\rangle \simeq\left\langle\left[\left(\nabla^{2}+q^{2}\right)\left(S^{-1} \not \beta\right)\right]\left[\left(\nabla^{2}+q^{2}\right)(\beta S)\right]\right\rangle,
\end{gathered}
$$

where $\not \equiv \equiv \sum_{i=1}^{3} \beta_{i} L_{i}$.

\section{APPENDIX C: PROPAGATORS}

The following propagators are derived from Eq. (17):

$$
\begin{aligned}
& \left(\begin{array}{cc}
\left\langle\delta(k) \delta^{*}(k)\right\rangle & \left\langle\delta(k) \Delta \beta_{3}^{*}(k)\right\rangle \\
\left\langle\Delta \beta_{3}(k) \delta^{*}(k)\right\rangle & \left\langle\Delta \beta_{3}(k) \Delta \beta_{3}^{*}(k)\right\rangle
\end{array}\right) \\
& \quad=\frac{1}{2} \frac{1}{S_{\delta 0}^{-1}(k) S_{0}^{-1}(k)+g^{2}(k)}\left(\begin{array}{cc}
S_{0}^{-1}(k) & g(k) \\
-g(k) & S_{\delta 0}^{-1}(k)
\end{array}\right),
\end{aligned}
$$

$$
\begin{aligned}
& \left(\begin{array}{cc}
\left\langle\Delta \beta_{i}(k) \Delta \beta_{i}^{*}(k)\right\rangle & \left\langle\Delta \beta_{i}(k) \Delta \beta_{i}^{*}(k+2 q \hat{z})\right\rangle \\
\left\langle\Delta \beta_{i}(k+2 q \hat{z}) \Delta \beta_{i}^{*}(k)\right\rangle & \left\langle\Delta \beta_{i}(k+2 q \hat{z}) \Delta \beta_{i}^{*}(k+2 q \hat{z})\right\rangle
\end{array}\right) \\
& =\frac{1}{2} \frac{1}{S_{0}^{-1}(k+2 q \hat{z}) S_{0}^{-1}(k)-G^{2}(k)} \\
& \quad \times\left(\begin{array}{cc}
S_{0}^{-1}(k+2 q \hat{z}) & -G(k) \\
-G(k) & S_{0}^{-1}(k)
\end{array}\right) .
\end{aligned}
$$

Here note that each component of the latter matrix $S(k)$ corresponds to summing all tree diagrams of the absorption/emission processes, for instance, the $\{1,1\}$ component reads

$$
\begin{aligned}
S_{11}(k) & =S_{0}(k) \sum_{n=0}^{\infty}\left[G(k) S_{0}(k+2 q \hat{z}) G(k) S_{0}(k)\right]^{n} \\
& =\frac{S_{0}^{-1}(k+2 q \hat{z})}{S_{0}^{-1}(k+2 q \hat{z}) S_{0}^{-1}(k)-G^{2}(k)} .
\end{aligned}
$$

\section{APPENDIX D: FLUCTUATION EFFECTS ON THE ORDER PARAMETER}

In averaging the order parameter over quadratic fluctuations in Eq. (22), we have used following results:

$$
\begin{aligned}
\left\langle\cos \left(q z+\beta_{3}\right)\right\rangle & =\cos q z \sum_{n=0}^{\infty} \frac{(-1)^{n}}{(2 n) !}\left\langle\beta_{3}^{2 n}\right\rangle \\
& =\cos q z e^{-\left\langle\beta_{3}^{2}\right\rangle / 2},
\end{aligned}
$$




$$
\begin{aligned}
\left\langle\delta \cos \left(q z+\beta_{3}\right)\right\rangle= & -\sin q z \\
& \times \sum_{n=0}^{\infty} \frac{(-1)^{n}}{(2 n+1) !}\left\langle\beta_{3}^{2 n}\right\rangle(2 n+1)\left\langle\delta \beta_{3}\right\rangle \\
= & -\sin q z\left\langle\delta \beta_{3}\right\rangle e^{-\left\langle\beta_{3}^{2}\right\rangle / 2},
\end{aligned}
$$

and similarly for the other contributions.

Also, the following finds useful in evaluating integrals like $\int \mathrm{d}^{3} k T / \omega_{ \pm}^{2}$,

$$
\int_{-\infty}^{\infty} \mathrm{d} k_{z} \frac{1}{a k_{z}^{2}+b+c k_{z}^{4}}=\frac{\pi}{2 A c}\left(\frac{1}{B_{-}}-\frac{1}{B_{+}}\right),
$$

where $(\tilde{a}, \tilde{b})=(a, b) / c, A^{2} \equiv \frac{\tilde{a}^{2}}{4}-\tilde{b}>0$, and $B_{ \pm}^{2} \equiv \frac{\tilde{a}}{2} \pm A$. In the general case where $a=c_{1}+c_{2} k_{t}^{2}, b=c_{3} k_{t}^{4}$, and $c=1$, for small $k_{t}$, the above integral results in as follows:

$$
\frac{\pi}{2 A c}\left(\frac{1}{B_{-}}-\frac{1}{B_{+}}\right) \simeq \frac{\pi}{c_{1}}\left(\sqrt{\frac{c_{1}}{c_{3}}} \frac{1}{k_{t}^{2}}-\frac{1}{\sqrt{c_{1}}}\right),
$$

where

$$
A \simeq \frac{c_{1}}{2}+\frac{c_{2}}{2} k_{t}^{2}-\frac{c_{3}}{c_{1}} k_{t}^{4} \quad \text { and } \quad B_{ \pm}^{2} \simeq\left\{\begin{array}{l}
c_{1}+c_{2} k_{t}^{2} \\
\frac{c_{3}}{c_{1}} k_{t}^{4}
\end{array}\right.
$$

Here, in a case that $a=4 q^{2}+2 k_{t}^{2}, b=k_{t}^{4}$, and $c=1$, which leads to $\int d k_{z} \omega_{-}^{-2}$, the integral can be evaluated as $\pi / 2 q k_{t}^{2}$, where $k_{t}$ is the momentum in the $x-y$ plane.

Hereafter we consider the expectation values of second order fluctuations in Eq. (22).

\section{Second order fluctuations for $\delta$ and $\beta_{3}$}

We evaluate the second order fluctuations by considering infrared (IR) singularities, and in high-temperature and low-energy expansion.

First of all, the second order fluctuations for $\beta_{3}$ result in as follows:

$$
\begin{aligned}
S_{3}(k) \equiv & 2\left\langle\Delta \beta_{3}(k) \Delta \beta_{3}^{*}(k)\right\rangle=\frac{S_{\delta 0}^{-1}(k)}{S_{\delta 0}^{-1}(k) S_{0}^{-1}(k)+g^{2}(k)} \\
= & \frac{\omega_{+}^{2}-\omega_{\delta}^{2}}{\omega_{+}^{2}-\omega_{-}^{2}} \frac{1}{\omega^{2}-\omega_{+}^{2}}+\frac{\omega_{\delta}^{2}-\omega_{-}^{2}}{\omega_{+}^{2}-\omega_{-}^{2}} \frac{1}{\omega^{2}-\omega_{-}^{2}} \\
\simeq & \frac{1}{\omega^{2}-\omega_{-}^{2}} \text { for small }|\vec{k}|, \\
S_{3}(\tau, \vec{k})= & T \sum_{n} e^{-i \omega_{n} \tau} S_{3}(k) \\
= & \frac{\omega_{+}^{2}-\omega_{\delta}^{2}}{\omega_{+}^{2}-\omega_{-}^{2}} \frac{n\left(\omega_{+}\right) e^{\omega_{+} \tau}+\left(n\left(\omega_{+}\right)+1\right) e^{-\omega_{+} \tau}}{2 \omega_{+}} \\
& +\frac{\omega_{\delta}^{2}-\omega_{-}^{2}}{\omega_{+}^{2}-\omega_{-}^{2}} \frac{n\left(\omega_{-}\right) e^{\omega_{-} \tau}+\left(n\left(\omega_{-}\right)+1\right) e^{-\omega_{-} \tau}}{2 \omega_{-}},
\end{aligned}
$$

$$
S_{3}(0, \vec{k}) \simeq \frac{T}{\omega_{-}^{2}} \quad \text { for small }|\vec{k}| \quad \text { and } \quad \omega_{ \pm} / T,
$$

where $\quad \omega_{\delta}^{2}=M^{2}+a_{6.4} \Delta^{2} \vec{k}^{2}+4 a_{6.1} q^{2}\left(k_{z}\right)^{2}+a_{6.1}\left(\vec{k}^{2}\right)^{2}$, and $n(x)=\left(e^{x / T}-1\right)^{-1}$ the Bose distribution function. Here the $T=0$ limit of (D6) reads $S_{3}(0, \vec{k}) \simeq \frac{1}{2 \omega_{-}}$.

Second, the second order fluctuations for $\delta$ result in as follows:

$$
\begin{aligned}
S_{\delta}(k) & \equiv 2\left\langle\delta(k) \delta^{*}(k)\right\rangle=\frac{S_{0}^{-1}(k)}{S_{\delta 0}^{-1}(k) S_{0}^{-1}(k)+g^{2}(k)} \\
& =\frac{\omega_{+}^{2}-\omega_{\beta}^{2}}{\omega_{+}^{2}-\omega_{-}^{2}} \frac{1}{\omega^{2}-\omega_{+}^{2}}+\frac{\omega_{\beta}^{2}-\omega_{-}^{2}}{\omega_{+}^{2}-\omega_{-}^{2}} \frac{1}{\omega^{2}-\omega_{-}^{2}} \\
& \simeq \frac{1}{\omega^{2}-\omega_{+}^{2}} \text { for small }|\vec{k}|,
\end{aligned}
$$

$S_{\delta}(\tau, \vec{k})=T \sum_{n} e^{-i \omega_{n} \tau} S_{\delta}(k)$

$$
\begin{aligned}
= & \frac{\omega_{+}^{2}-\omega_{\beta}^{2}}{\omega_{+}^{2}-\omega_{-}^{2}} \frac{n\left(\omega_{+}\right) e^{\omega_{+} \tau}+\left(n\left(\omega_{+}\right)+1\right) e^{-\omega_{+} \tau}}{2 \omega_{+}} \\
+ & \frac{\omega_{\beta}^{2}-\omega_{-}^{2}}{\omega_{+}^{2}-\omega_{-}^{2}} \frac{n\left(\omega_{-}\right) e^{\omega_{-} \tau}+\left(n\left(\omega_{-}\right)+1\right) e^{-\omega_{-} \tau}}{2 \omega_{-}}, \\
& S_{\delta}(0, \vec{k}) \simeq \frac{T}{\omega_{+}^{2}} \text { for small }|\vec{k}| \text { and } \omega_{ \pm} / T,
\end{aligned}
$$

where $\omega_{\beta}^{2}=a_{6,1}\left[4 q^{2} k_{z}^{2}+\left(\vec{k}^{2}\right)^{2}\right]$, and the $T=0$ limit of (D8) $\operatorname{reads} S_{3}(0, \vec{k}) \simeq \frac{1}{2 \omega_{+}}$.

Finally, the second order fluctuations for $\delta$ and $\beta_{3}$ result in as follows:

$$
\begin{aligned}
S_{\delta 3}(k) \equiv & 2\left\langle\delta(k) \Delta \beta_{3}^{*}(k)\right\rangle=\frac{g(k)}{S_{\delta 0}^{-1}(k) S_{0}^{-1}(k)+g^{2}(k)} \\
= & \frac{g(k)}{\omega_{+}^{2}-\omega_{-}^{2}}\left(\frac{1}{\omega^{2}-\omega_{+}^{2}}-\frac{1}{\omega^{2}-\omega_{-}^{2}}\right), \\
S_{\delta 3}(\tau, \vec{k})= & T \sum_{n} e^{-i \omega_{n} \tau} S_{\delta 3}(k) \\
= & \frac{g(k)}{\omega_{+}^{2}-\omega_{-}^{2}}\left[\frac{n\left(\omega_{-}\right) e^{\omega_{-} \tau}+\left(n\left(\omega_{-}\right)+1\right) e^{-\omega_{-} \tau}}{2 \omega_{-}}\right. \\
& \left.-\frac{n\left(\omega_{+}\right) e^{\omega_{+} \tau}+\left(n\left(\omega_{+}\right)+1\right) e^{-\omega_{+} \tau}}{2 \omega_{+}}\right], \quad(\mathrm{D} 10) \\
S_{\delta 3}(0, \vec{k}) \simeq & \frac{g(k)}{\omega_{+}^{2}-\omega_{-}^{2}}\left[\frac{T}{\omega_{-}^{2}}-\frac{T}{\omega_{+}^{2}}\right] \\
\simeq & \frac{2 i q \Delta^{2} a_{6.2} k_{z}}{M^{2}} \frac{T}{\omega_{-}^{2}} \quad \text { for small }|\vec{k}| \quad \text { and } \quad \omega_{ \pm} / T .
\end{aligned}
$$

From the above results, the second order fluctuations for $\delta$ and $\beta_{3}$ in real space read $\Delta^{2}\left\langle\beta_{3}^{2}(x)\right\rangle=\frac{1}{2} \int \frac{\mathrm{d}^{3} k}{(2 \pi)^{3}} S_{3}(0 ; \vec{k}) \simeq$ $\frac{1}{2} \int \frac{\mathrm{d}^{3} k}{(2 \pi)^{3}} \frac{T}{\omega_{-}^{2}}$, and $\Delta\left\langle\delta(x) \beta_{3}(x)\right\rangle=\frac{1}{2} \int \frac{\mathrm{d}^{3} k}{(2 \pi)^{3}} S_{\delta 3}(0 ; \vec{k}) \simeq 0$. 
LEE et al.

\section{Second order fluctuations for $\beta_{1}$ and $\beta_{2}$}

Here for later convenience, we calculate the imaginary time correlations of mean square fluctuation of the $\beta_{1,2}$.

For the $\beta_{1,2}$ with same momentum,

$$
\begin{gathered}
S_{11}(k) \equiv 2\left\langle\Delta \beta_{1,2}(k) \Delta \beta_{1,2}^{*}(k)\right\rangle=\frac{S_{0}^{-1}(k+2 q \hat{z})}{S_{0}^{-1}(k+2 q \hat{z}) S_{0}^{-1}(k)-G^{2}(k)}, \\
S_{11}(\tau, \vec{k})=T \sum_{n} e^{-i \omega_{n} \tau} S_{11}(k)=\frac{f_{1}(\vec{k})}{2 \omega_{k}}\left[n\left(\omega_{k}\right) e^{\omega_{k} \tau}+\left(n\left(\omega_{k}\right)+1\right) e^{-\omega_{k} \tau}\right], \\
S_{11}(0, \vec{k}) \simeq \frac{T}{\omega_{k}^{2}} \quad \text { for small }|\vec{k}| \quad \text { and } \quad \omega_{k} / T,
\end{gathered}
$$

where $\omega_{k}$ is the dispersion relation for $\beta_{1,2}$ given by Eq. (20), and $f_{1}(\vec{k})=\left(1-\frac{Y_{+1}-Y_{-1}}{Y_{+3}-Y_{+1}}\right)^{-2} \simeq 1$ for $|\vec{k}| \simeq 0$ with $Y_{ \pm n}:=\left(\vec{k}^{2}-q^{2}\right)_{k_{z} \rightarrow k_{z}+n q}^{2}$. Here the $T=0$ limit of (D12) reads $S_{11}(0, \vec{k}) \simeq \frac{1}{2 \omega_{k}}$.

Similarly, for the $\beta_{1,2}$ with different momentum,

$$
\begin{gathered}
S_{12}(k) \equiv 2\left\langle\Delta \beta_{1,2}(k) \Delta \beta_{1,2}^{*}(k+2 q \hat{z})\right\rangle=\frac{-G(k)}{S_{0}^{-1}(k+2 q \hat{z}) S_{0}^{-1}(k)-G^{2}(k)}, \\
S_{12}(\tau, \vec{k})=T \sum_{n} e^{-i \omega_{n} \tau} S_{12}(k)=\frac{f_{2}(\vec{k})}{2 \omega_{k}}\left[n\left(\omega_{k}\right) e^{\omega_{k} \tau}+\left(n\left(\omega_{k}\right)+1\right) e^{-\omega_{k} \tau}\right], \\
S_{12}(0, \vec{k}) \simeq \frac{k_{z} \vec{k}^{2}}{8 q^{3}} \frac{T}{\omega_{k}^{2}} \quad \text { for small }|\vec{k}| \quad \text { and } \quad \omega_{k} / T,
\end{gathered}
$$

where $f_{2}(\vec{k})=\frac{Y_{+1}-Y_{-1}}{Y_{+3}-Y_{+1}}\left(1-\frac{Y_{+1}-Y_{-1}}{Y_{+3}-Y_{+1}}\right)^{-2} \simeq \frac{k_{z} \vec{k}^{2}}{8 q^{3}}$ for $|\vec{k}| \simeq 0$. Here $S_{12}(0, \vec{k}) \simeq \frac{k_{z} \vec{k}^{2}}{8 q^{3}} \frac{1}{2 \omega_{k}}$ for $T=0$ limit of (D14).

From the above results, the real space fluctuation reads

$$
\begin{aligned}
\Delta^{2}\left\langle\beta_{i=1,2}^{2}(x)\right\rangle= & \sum \int \mathrm{d} p \mathrm{~d} k e^{i(p+k) x}\left\langle\Delta \beta_{i}(p) \Delta \beta_{i}(k)\right\rangle \\
= & 2 \cos 2 q z \sum \mathrm{d} k \operatorname{Re}\left\langle\Delta \beta_{i}^{*}(k+2 q \hat{z}) \Delta \beta_{i}(k)\right\rangle+2 \sin 2 q z \sum \mathrm{d} k \operatorname{Im}\left\langle\Delta \beta_{i}^{*}(k+2 q \hat{z}) \Delta \beta_{i}(k)\right\rangle \\
& +\oint \mathrm{d} k\left\langle\Delta \beta_{i}(-k) \Delta \beta_{i}(k)\right\rangle \\
= & 2 \cos 2 q z \int \frac{\mathrm{d}^{3} k}{(2 \pi)^{3}} \frac{S_{21}(0 ; \vec{k})}{2}+\int \frac{\mathrm{d}^{3} k}{(2 \pi)^{3}} \frac{S_{11}(0 ; \vec{k})}{2},
\end{aligned}
$$

where in the last line the first term gives zero due to the odd function, and the second diverges logarithmically at $k=0$ at finite temperature.

\section{APPENDIX E: ORDER PARAMETER CORRELATIONS}

The following integral is useful in evaluation of order parameter correlation functions at long range,

$$
\int_{-\infty}^{\infty} \mathrm{d} k_{z} \frac{\cos \left(k_{z} z\right)}{a k_{z}^{2}+b+c k_{z}^{4}}=\frac{\pi}{2 c A}\left(\frac{e^{-B_{-} z}}{B_{-}}-\frac{e^{-B_{+} z}}{B_{+}}\right)
$$

where $A^{2} \equiv \frac{\tilde{a}^{2}}{4}-\tilde{b}>0, B_{ \pm}^{2} \equiv \frac{\tilde{a}}{2} \pm A$, and $(\tilde{a}, \tilde{b})=(a, b) / c$. In general case where $a=c_{1}+c_{2} k_{t}^{2}, b=c_{3} k_{t}^{4}$, for small $k_{t}$, the above integral results in as follows: 


$$
\frac{\pi}{2 c A}\left(\frac{e^{-B_{-} z}}{B_{-}}-\frac{e^{-B_{+} z}}{B_{+}}\right) \simeq \frac{\pi}{c_{1}}\left(\frac{e^{-\sqrt{\frac{c_{3}}{c_{1}}} k_{t}^{2} z}}{\sqrt{\frac{c_{3}}{c_{1}}} k_{t}^{2}}-\frac{e^{-\sqrt{\frac{c_{1}}{c} z}}}{\sqrt{\frac{c_{1}}{c}}}\right),
$$

where

$$
A \simeq \frac{\tilde{c}_{1}}{2}+\frac{\tilde{c}_{2}}{2} k_{t}^{2}-\frac{\tilde{c}_{3}}{\tilde{c}_{1}} k_{t}^{4} \quad \text { and } \quad B_{ \pm}^{2} \simeq\left\{\begin{array}{l}
\tilde{c}_{1}+\tilde{c}_{2} k_{t}^{2} \\
\frac{\tilde{c}_{3}}{\tilde{c}_{1}} k_{t}^{4}
\end{array}\right.
$$

With the above results, we can evaluate the following integral,

$$
\begin{aligned}
& \frac{1}{(2 \pi)^{2}} \int_{-\infty}^{\infty} \mathrm{d} k_{z} \int_{0}^{\Lambda} \mathrm{d} k_{t} k_{t} \frac{1-\cos \left(k_{z} z\right)}{\left(c_{1}+c_{2} k_{t}^{2}\right) k_{z}^{2}+c_{3} k_{t}^{4}+c k_{z}^{4}} \\
& \quad \int_{0}^{\Lambda} \frac{\mathrm{d} k_{t} k_{t}}{(2 \pi)^{2}} \frac{\pi}{c_{1}}\left[\left(\frac{1}{\sqrt{\frac{c_{3}}{c_{1}}} k_{t}^{2}}-\frac{1}{\sqrt{\frac{c_{1}}{c}}}\right)-\left(\frac{e^{-\sqrt{\frac{c_{3}}{c_{1}}} k_{t}^{2} z}}{\sqrt{\frac{c_{3}}{c_{1}}} k_{t}^{2}}-\frac{e^{-\sqrt{\frac{c_{1}}{c}} z}}{\sqrt{\frac{c_{1}}{c}}}\right)\right] \quad \text { for IR region of } k_{t} \\
& \quad \int_{0}^{\Lambda} \frac{\mathrm{d} k_{t}}{4 \pi} \frac{1-e^{-\sqrt{\frac{c_{3}}{c_{1}}} k_{t}^{2} z}}{c_{1} \sqrt{\frac{c_{3}}{c_{1}}} k_{t}} \quad \text { for large } z \simeq \frac{1}{8 \sqrt{c_{1} c_{3}} \pi} \ln \left(\sqrt{\frac{c_{3}}{c_{1}}} z \Lambda^{2}\right) .
\end{aligned}
$$

where $\Lambda$ is the ultraviolet cutoff.

Similarly, the following integral is also useful,

$$
\int_{-\infty}^{\infty} \mathrm{d} k_{z} \frac{\sin \left(k_{z} z\right) k_{z}}{a k_{z}^{2}+b+c k_{z}^{4}}=\frac{\pi}{2 c A}\left(e^{-B_{-} z}-e^{-B_{+} z}\right) .
$$

In general case where $a=c_{1}+c_{2} k_{t}^{2}, b=c_{3} k_{t}^{4}$, for small $k_{t}$, we obtain

$$
\frac{\pi}{2 c A}\left(e^{-B_{-} z}-e^{-B_{+} z}\right) \simeq \frac{\pi}{c_{1}}\left(e^{-\sqrt{\frac{c_{3}}{c_{1}} k_{t}^{2} z}}-e^{-\sqrt{\frac{c_{1}}{c} z}}\right) .
$$

With this result, we can evaluate the following integral,

$$
\begin{aligned}
\frac{2 \pi}{(2 \pi)^{3}} \int_{-\infty}^{\infty} \mathrm{d} k_{z} \int_{0}^{\Lambda} \mathrm{d} k_{t} k_{t} \frac{\sin \left(k_{z} z\right) k_{z}}{a k_{z}^{2}+b+c k_{z}^{4}} & \simeq \int_{0}^{\Lambda} \frac{\mathrm{d} k_{t} k_{t}}{(2 \pi)^{2}} \frac{\pi}{c_{1}}\left(e^{-\sqrt{\frac{c_{3}}{c_{1}}} k_{t}^{2} z}-e^{-\sqrt{\frac{c_{1}}{c}} z}\right) \text { for IR region of } k_{t} \\
& \simeq \int_{0}^{\Lambda} \frac{\mathrm{d} k_{t}}{4 \pi c_{1}} k_{t} e^{-\sqrt{\frac{c_{3}}{c_{1}}} k_{t}^{2} z} \text { for large } z \\
& \simeq \frac{1}{8 \pi \sqrt{c_{1} c_{3}}} \frac{1}{z} .
\end{aligned}
$$

\section{Long-range correlations of diagonal components}

In evaluating the long-range correlation functions of diagonal components (29), the following expectation values with $\beta_{i}^{ \pm} \equiv \beta_{i}(z) \pm \beta_{i}(0)$ are useful:

$$
\begin{gathered}
\left\langle\cos \left(q z+\beta_{i=1,2,3}^{ \pm}\right)\right\rangle=\cos q z e^{-\left\langle\beta_{i}^{ \pm 2}\right\rangle / 2}, \\
\left\langle\delta(x) \cos \left(q z+\beta_{3}^{ \pm}\right)\right\rangle=\mp \sin q z\left\langle\delta(x) \beta_{3}(0)\right\rangle e^{-\left\langle\beta_{3 \pm}^{2}\right\rangle / 2}, \\
\left\langle\delta(0) \cos \left(q z+\beta_{3}^{ \pm}\right)\right\rangle=-\sin q z\left\langle\delta(0) \beta_{3}(x)\right\rangle e^{-\left\langle\beta_{3 \pm}^{2}\right\rangle / 2},
\end{gathered}
$$


LEE et al.

$$
\left\langle\delta(x) \delta(0) \cos \left(q z+\beta_{3}^{ \pm}\right)\right\rangle=\cos q z e^{-\left\langle\beta_{3 \pm}^{2}\right\rangle / 2}\left[\langle\delta(x) \delta(0)\rangle \mp\left\langle\delta(x) \beta_{3}(0)\right\rangle\left\langle\delta(0) \beta_{3}(x)\right\rangle\right],
$$

where

$$
\begin{aligned}
\Delta\left\langle\delta(x) \beta_{3}(0)\right\rangle= & \Delta \int \mathrm{d} k e^{i k x}\left\langle\delta(k) \beta_{3}(-k)\right\rangle \\
= & \frac{1}{2} \int \frac{\mathrm{d}^{3} k}{(2 \pi)^{3}} e^{i \vec{k} \cdot \vec{x}} S_{\delta 3}(0 ; \vec{k}) \\
\simeq & \frac{1}{2} \int \frac{\mathrm{d}^{3} k}{(2 \pi)^{3}} i \sin \left(k_{z} z\right) \frac{2 i q \Delta^{2} a_{6.2} k_{z}}{M^{2}} \frac{T}{\omega_{-}^{2}} \quad \text { for } x \| \hat{z} \\
\simeq & -\frac{a_{6.2} q \Delta^{2} T}{8 \pi a_{6.1} M^{2}} \frac{1}{q z}, \\
\left\langle\beta_{3}^{ \pm 2}\right\rangle / 2= & \sum \frac{\left.\mathrm{d} k(1) \beta_{3}(x)\right\rangle=-\left\langle\delta(x) \beta_{3}(0)\right\rangle,}{\left.i k_{z} z\right)\left\langle\beta_{3}(k) \beta_{3}^{*}(k)\right\rangle} \\
= & \frac{1}{2 \Delta^{2}} \int \frac{\mathrm{d}^{3} k}{(2 \pi)^{3}}\left(1 \pm \cos k_{z} z\right) S_{3}(0 ; \vec{k}) \\
& \simeq \frac{T}{2 \Delta^{2}} \int \frac{\mathrm{d}^{3} k}{(2 \pi)^{3}} \frac{1 \pm \cos k_{z} z}{\omega_{-}^{2}} \\
& =\left\{\begin{array}{l}
\infty \\
\sim \frac{T}{16 \pi a_{6,1} \Delta^{2} u_{z-}} \ln \frac{z \Lambda^{2}}{2 q},
\end{array}\right.
\end{aligned}
$$

and

$$
\begin{aligned}
\left\langle\beta_{i=1,2}^{ \pm}\right\rangle / 2 & =\sum \mathrm{d} k\left[\left\langle\beta_{i}(k) \beta_{i}^{*}(k)\right\rangle\left(1 \pm e^{i k_{z} z}\right) \pm\left(\left\langle\beta_{i}(k) \beta_{i}^{*}(k+2 q \hat{z})\right\rangle e^{i k_{z} z}+\text { c.c. }\right)\right] \\
& =\frac{1}{2 \Delta^{2}} \int \frac{\mathrm{d}^{3} k}{(2 \pi)^{3}}\left[\left(1 \pm \cos k_{z} z\right) S_{11}(0 ; \vec{k}) \pm\left(\sin k_{z} z \operatorname{Im} S_{12}(0 ; \vec{k})+\cos k_{z} z \operatorname{Re} S_{12}(0 ; \vec{k})\right)\right] \\
& \simeq \frac{T}{2 \Delta^{2}} \int \frac{\mathrm{d}^{3} k}{(2 \pi)^{3}} \frac{1 \pm \cos k_{z} z}{\omega_{k}^{2}} \\
& =\left\{\begin{array}{l}
\infty \\
\sim \frac{T}{16 \pi a_{6,1} \Delta^{2} u_{z-}} \ln \frac{z \Lambda^{2}}{2 q}
\end{array}\right.
\end{aligned}
$$

From the above results, for instance, the $\{1,1\}$ component in the $z$ direction reads

$$
\begin{aligned}
f_{11}(\hat{z} z) & =\left\langle(\Delta+\delta(z))(\Delta+\delta(0)) \cos \left(q z+\beta_{3}(z)\right) \cos \beta_{3}(0) \cos \beta_{2}(z) \cos \beta_{2}(0) \cos \beta_{1}(z) \cos \beta_{1}(0)\right\rangle \\
& =\frac{1}{8}\left\langle(\Delta+\delta(z))(\Delta+\delta(0))\left[\cos \left(q z+\beta_{3}^{+}\right)+\cos \left(q z+\beta_{3}^{-}\right)\right]\right\rangle\left\langle\left(\cos \beta_{2}^{+}+\cos \beta_{2}^{-}\right)\left(\cos \beta_{1}^{+}+\cos \beta_{1}^{-}\right)\right\rangle \\
& \simeq \frac{1}{8} e^{-\sum_{i}\left\langle\beta_{i}^{-2}\right\rangle / 2}\left[\Delta^{2} \cos q z\left(1-\left\langle\delta(z) \beta_{3}(0)\right\rangle^{2}\right)+2 \Delta \sin q z\left\langle\delta(z) \beta_{3}(0)\right\rangle\right] \\
& \simeq \frac{1}{8} \Delta^{2} \cos q z e^{-\sum_{i=1,2,3}\left\langle\beta_{i}^{-2}\right\rangle / 2},
\end{aligned}
$$

where we have used the fact that $\left\langle\beta_{i}^{+2}\right\rangle$ are logarithmically divergent, and terms including $\left\langle\delta(z) \beta_{3}(0)\right\rangle \propto z^{-1}$ drop faster than others for a large distance in the $z$ direction. Also, $\langle\delta(z) \delta(0)\rangle$ corresponds to a massive mode, which does not contribute to the long-range correlations. 


\section{LANDAU-PEIERLS INSTABILITY IN A FULDE-FERRELL ...}

Similarly, in the $x-y$ directions, we can obtain the following results:

$$
\begin{gathered}
\left\langle\cos \beta_{i=1,2,3}^{ \pm}\right\rangle=e^{-\left\langle\beta_{i}^{ \pm 2}\right\rangle / 2}, \\
\left\langle\delta\left(x_{t}\right) \cos \beta_{3}^{ \pm}\right\rangle=0, \\
\left\langle\delta(0) \cos \beta_{3}^{ \pm}\right\rangle=0, \\
\left\langle\delta\left(x_{t}\right) \delta(0) \cos \beta_{3}^{ \pm}\right\rangle=e^{-\left\langle\beta_{3 \pm}^{2}\right\rangle / 2}\left[\left\langle\delta\left(x_{t}\right) \delta(0)\right\rangle \mp\left\langle\delta\left(x_{t}\right) \beta_{3}(0)\right\rangle\left\langle\delta(0) \beta_{3}\left(x_{t}\right)\right\rangle\right],
\end{gathered}
$$

where $\beta_{i}^{ \pm} \equiv \beta_{i}\left(x_{t}\right) \pm \beta_{i}(0)$, and

$$
\left\langle\beta_{i=1,2,3}^{ \pm^{2}}\right\rangle / 2=\left\{\begin{array}{l}
\infty \\
\sim \frac{T}{16 \pi a_{6,1} \Delta^{2} u_{z-}} \ln \left(x_{t} \Lambda\right)^{2} .
\end{array}\right.
$$

[1] K. Fukushima and T. Hatsuda, Rep. Prog. Phys. 74, 014001 (2011); K. Fukushima and C. Sasaki, Prog. Part. Nucl. Phys. 72, 99 (2013).

[2] B. Friman, C. Höhne, J. Knoll, S. Leupold, J. Randrup, R. Rapp, and P. Senger, Lect. Notes Phys. 814, 11 (2011).

[3] M. Buballa and S. Carignano, Prog. Part. Nucl. Phys. 81, 39 (2015).

[4] E. Nakano and T. Tatsumi, Phys. Rev. D 71, 114006 (2005).

[5] D. Nickel, Phys. Rev. D 80, 074025 (2009).

[6] D. Müller, M. Buballa, and J. Wambach, Phys. Lett. B 727, 240 (2013).

[7] D. Nickel, Phys. Rev. Lett. 103, 072301 (2009).

[8] S. Carignano, M. Buballa, and B.-J. Schaefer, Phys. Rev. D 90, 014033 (2014).

[9] D. V. Deryagin, D. Y. Grigoriev, and V. A. Rubakov, Int. J. Mod. Phys. A 07, 659 (1992).

[10] E. Shuster and D. T. Son, Nucl. Phys. B573, 434 (2000).

[11] L. McLerran and R. D. Pisarski, Nucl. Phys. A796, 83 (2007).

[12] T. Kojo, Y. Hidaka, L. McLerran, and R. D. Pisarski, Nucl. Phys. A843, 37 (2010); T. Kojo, Y. Hidaka, K. Fukushima, L. D. McLerran, and R. D. Pisarski, Nucl. Phys. A875, 94 (2012).

[13] V. Schon and M. Thies, in At the Frontier of Particle Physics, edited by M. Shifman (World Scientific, Singapore, 2001), Vol. 3, p. 1945; M. Thies, J. Phys. A 39, 12707 (2006); G. Basar, G. V. Dunne, and M. Thies, Phys. Rev. D 79, 105012 (2009).

[14] H. Abuki, D. Ishibashi, and K. Suzuki, Phys. Rev. D 85, 074002 (2012).

[15] S. Carignano and M. Buballa, Phys. Rev. D 86, 074018 (2012).

[16] P. Fulde and A. Ferrell, Phys. Rev. 135, A550 (1964).
[17] A. Larkin and Y. Ovchinnikov, Sov. Phys. JETP 20, 762 (1965).

[18] F. Dautry and E. M. Nyman, Nucl. Phys. A319, 323 (1979).

[19] L. D. Landau and E. M. Lifshitz, Statistical Physics (Pergamon Press, Oxford, 1969).

[20] G. Baym, B. L. Friman, and G. Grinstein, Nucl. Phys. B210, 193 (1982).

[21] J. Als-Nielsen, R. J. Birgeneau, M. Kaplan, J. D. Litster, and C. R. Safinya, Phys. Rev. Lett. 39, 1668 (1977); J. Als-Nielsen, J. D. Litster, R. J. Birgeneau, M. Kaplan, C. R. Safinya, A. Lindegaard-Andersen, and S. Mathiesen, Phys. Rev. B 22, 312 (1980).

[22] P. G. de Gennes and J. Prost, The Physics of Liquid Crystals, (Oxford University Press, New York, 1993); P. M. Chaikin and T.C. Lubensky, Principles of Condensed Matter Physics (Cambridge University Press, Cambridge, England, 2000).

[23] H. Shimahara, J. Phys. Soc. Jpn. 67, 1872 (1998); H. Shimahara, Physica B (Amsterdam) 259-261, 492 (1999); Y. Ohashi, J. Phys. Soc. Jpn. 71, 2625 (2002).

[24] L. Radzihovsky and A. Vishwanath, Phys. Rev. Lett. 103, 010404 (2009); L. Radzihovsky, Phys. Rev. A 84, 023611 (2011); Physica C (Amsterdam) 481, 189 (2012).

[25] B. W. Lee, Chiral Dynamics (Gordon and Breach, New York, 1972).

[26] M. Kobayashi and M. Nitta, Phys. Rev. Lett. 113, 120403 (2014).

[27] Y. Hidaka, T. Noumi, and G. Shiu, arXiv:1412.5601 [Phys. Rev. D (to be published)].

[28] I. Low and A. V. Manohar, Phys. Rev. Lett. 88, 101602 (2002); H. Watanabe and H. Murayama, Phys. Rev. Lett. 110, 181601 (2013); T. Hayata and Y. Hidaka, Phys. Lett. B 735, 195 (2014). 
LEE et al.

[29] V. L. Berezinsky, Sov. Phys. JETP 32, 493 (1971); 34, 610 (1972); J. M. Kosterlitz and D. J. Thouless, J. Phys. C 6, 1181 (1973); J. M. Kosterlitz, J. Phys. C 7, 1046 (1974).

[30] T. Nattermann, Phys. Rev. Lett. 64, 2454 (1990); T. Giamarchi and P. Le Doussal, Phys. Rev. Lett. 72, 1530 (1994); Phys. Rev. B 52, 1242 (1995).

[31] W. H. Nitsche, N. Y. Kim, G. Roumpos, C. Schneider, M. Kamp, S. Höfling, A. Forchel, and Y. Yamamoto, Phys. Rev. B 90, 205430 (2014).

[32] S. Carignano, D. Nickel, and M. Buballa, Phys. Rev. D 82, 054009 (2010).

[33] I. E. Frolov, V. C. Zhukovsky, and K. G. Klimenko, Phys. Rev. D 82, 076002 (2010).

[34] T. Tatsumi, K. Nishiyama, and S. Karasawa, Phys. Lett. B 743, 66 (2015).

[35] G. Watanabe, K. Iida, and K. Sato, Nucl. Phys. A676, 455 (2000); K. Nakazato, K. Oyamatsu, and S. Yamada,
PHYSICAL REVIEW D 92, 034024 (2015)

Phys. Rev. Lett. 103, 132501 (2009); T. Maruyama, G. Watanabe, and S. Chiba, Prog. Theor. Exp. Phys. 2012, 01A201 (2012); B. Schuetrumpf, M. A. Klatt, K. Iida, J. A. Maruhn, K. Mecke, and P.-G. Reinhard, Phys. Rev. C 87, 055805 (2013); M. Okamoto, T. Maruyama, K. Yabana, and T. Tatsumi, Phys. Rev. C 88, 025801 (2013).

[36] N. Yasutake, T. Maruyama, and T. Tatsumi, Phys. Rev. D 80, 123009 (2009); 86, 101302 (2012); N. Yasutake, R. Łastowiecki, S. Benić, D. Blaschke, T. Maruyama, and T. Tatsumi, Phys. Rev. C 89, 065803 (2014).

[37] T. Tatsumi and T. Muto, Phys. Rev. D 89, 103005 (2014).

[38] S. Carignano, E. J. Ferrer, V. de la Incera, and L. Paulucci, arXiv:1505.05094.

[39] H. Abuki, Phys. Rev. D 87, 094006 (2013); Phys. Lett. B 728, 427 (2014).

[40] J. Moreira, B. Hiller, W. Broniowski, A. A. Osipov, and A. H. Blin, Phys. Rev. D 89, 036009 (2014). 